\title{
EXPERIMENTAL IMPLEMENTATION OF RAMAN SCATTERING SPECTROSCOPY, PHOTOLUMINESCENCE AND SOME OPTICAL PROPERTIES OF SILVER NANOPARTICLES CREATED BY ECO-FRIENDLY TECHNIQUE
}

\author{
Nadia Mohammed Jassim $\bowtie$ \\ College of Science ${ }^{l}$ \\ nadiajassim@uodiyala.edu.iq \\ Alyaa Hussein Ali \\ College of Science \\ Ammar Jassim Mohammed \\ Department of Chemistry \\ College of Education for Pure Sciences (Ibn Al-Haitham) \\ University of Baghdad \\ Baghdad city, Baghdad governorate, Iraq \\ ${ }^{1}$ University of Diyala \\ Baquba City, Daiyla governorate, Iraq, 32001
}

$\triangle$ Corresponding author

\begin{abstract}
The object of research is studying Raman scattering technique, photoluminescence and some optical properties of silver nanoparticles created by eco-friendly technique which independent on a long time, effort, energy and high temperatures, and with the highest adsorption capacity in order to achieve a high inhibition to paralyze the activity of the bacterial wall, by achieving the highest surface plasmon resonance (SRR). Silver nanoparticles were prepared using Matricaria Flower extract. Characterization of silver nanoparticles and detection of their effectiveness against microbial using two types of bacteria (Escherichia Coli and Staphylococcus aureus), these nanoparticles were measured using a number of measurements, X-ray diffraction measurement, Energy Dispersion (EDX), (FESEM), $U-V$ Spectroscopy, Fourier Transform Infrared (FTIR), Photoluminescence (PL) properties of silver nanoparticle at room temperature and Raman scattering spectroscopy were investigated. The Scherrer's equation was used to calculate the crystallite size of Silver nanoparticles, the average crystallite size is $48.64 \mathrm{~nm}$. The PL spectra of silver nanoparticles exhibit two emission bands: one is in the UV region $350 \mathrm{~nm}$ and the other is in the visible region $650 \mathrm{~nm}$. This is roughly identical to the absorption spectrum results. The antimicrobial activity was tested against gram negative bacteria (Escherichia coli) and gram positive bacteria (Staphylococcus aureus). Prepared Ag NPs exhibited inhibitory activity in both types of bacteria strains at best selectivity against gram-negative bacteria.

An eco-friendly technique is used for synthesizing technique to produce silver nanoparticles with the expected best application properties. These research results suitable to be use the Silver nanoparticles in sensors and many electronic, electrical, medical and biological applications. ly method.

Keywords: Raman scattering technique, Photoluminescence, Silver nanoparticles, Surface plasmon resonance, Eco-friend-
\end{abstract}

\section{Introduction}

Nano-science is known as the science of nanomaterials. It is a new field of researchers dealing with nanoparticles with a diameter of less than $100 \mathrm{~nm}$. Nanotechnology is widely used in medicinal chemistry, physics and other fields. Materials whose crystal size is less than $100 \mathrm{~nm}$ are called nanocrystals [1]. Nanotechnology has led to great advances in the field of biomedicine, like drug delivery, and check the structure of DNA [2]. Nanoparticles are great importance in nanotechnology, as it shows new properties of matter, its primary function is biological extermination, 
which adds to its antimicrobial properties [3]. These particles have many applications in many fields, including chemical stimulation, photosynthesis, antimicrobials, and detection of diseases, clothes, food packaging, anti- wounds, and biomedical products [4]. There are several methods for preparing silver nanoparticles, chemical method, physical and biological, and that the biological method is one of the best ways to synthesize silver nanoparticles, because it does not leave toxic substances, the fastest and the safest way, the materials obtained in this way are characterized by high purity, good electron conductivity, chemical stability, and high motivational effectiveness [5]. It is anti-fungal, anti- inflammatory and anti- virus studies have shown that it is difficult to completely remove silver if it is deposited in the body. and through the numerous studies conducted on humans and animals. It was found that nanosilver, can be excreted through hair, faces and urine significantly [6].

The paper [7] presents the results of research on «Phytomediated Photo-Induced Green Synthesis of Silver Nanoparticles Using Matricaria chamomilla L. and ITS CATALYTIC ACTIVITY against Rhodamine». The study of the bio-fabrication of silver nanoparticles (AgNPs) was carried out through the facile green route, using the aqueous extract of Matricaria chamomilla. But there were unresolved issues related to the studying of optical properties and some important application such as antibacterial activity. The reason for this may be the objective difficulties associated with the studying techniques, cost part in terms of device of studying techniques, which makes relevant research impractical, etc. A way to overcome these difficulties can be to use easy studying techniques. This approach was used in [2]. All this suggests that it is advisable to conduct a study on the cost of the studying techniques. The papers $[8,9]$ present the results of research on plant mediated synthesis of silver nanoparticles using some extracts plants The syntheses of Ag nanoparticles are shown. But there were unresolved issues related to the type of application used. The reason for this may be the objective difficulties associated with the type of applications, which makes relevant research impractical, etc. A way to overcome these difficulties can be to use specific application such as biological application and antibacterial activity. This approach was used in [10]. All this suggests that it is advisable to conduct a study on experimental studying of some optical properties of silver nanoparticles created by eco-friendly technique.

The relevance of the research goal is experimental implementation of Raman scattering spectroscopy, photoluminescence and studying of some optical properties of silver nanoparticles which synthesized from Matricaria flower extract by eco- friendly method that does not require much effort and long time. It is a safe, economical and inexpensive method. All these features made it one of the most desirable techniques for synthesizing nanoparticles such as silver nanoparticles. Raman scattering technique and photoluminescence experiments were carried out in order to study the optical emission of silver nanoparticles which arises from the Surface Plasmon Resonance (SPR) predicated on the collective oscillation of conduction electrons created by an electromagnetic field [7]. Let's expect these results will useful trial to use metal-enhanced fluorescence or Raman scattering spectra in different application such as biosensing and optoelectronics. The synthesized silver nanoparticles present antibacterial activity antimicrobial activities against some kinds of bacteria such as Escherichia coli and Staphylococcus aurous. Advantages of utilizing green synthesis is that it is energy effective, cost efficient, saving human health and surroundings. This green synthesis method could be considered instead of classical chemical and physical methods and therefore has a potential applications in many field such as biomedical and medical applications. The critical analysis of the existing problem, which the research is aimed at solving is synthesis of silver nanoparticles using extracts plants and studying of some their optical properties and using it as antibacterial activity. The direction should the study be developed is synthesis of silver nanoparticles by plant extracts has emerged as a promising field of research due to certain advantages compared to other methods. extract plant is regarded as a material promising to created and synthesis silver nanoparticles with economic and safe method and using it in study of optical properties with easy use in antibacterial activity.

\section{Materials and methods}

The experiment was conducted with the use of eco-friendly method. Matricaria flowers plant were used to synthesized silver nanoparticles. The morphology and structural analysis of the prepared silver nanoparticles has been carried out by XRD, FESEM, FTIR. The optical absor- 
bance spectra of silver colloidal solution was measured using a PerkinlEmer Lambda 1.1 UV-Vis Spectrphotometer.

The Raman experiments were carried out using a Joibin-Ywon XplorE setup. The excitation laser beam $532 \mathrm{~nm}$ was focused onto the sample through the 100X lens of a confocal microscope. The laser power was $150 \mathrm{mw}$ and the Raman spectra were measured with an accumulation time of $15 \mathrm{~s}$. The photoluminescence measurements were carried out at room temperature using a PerkinlEmer spectrophotometer (L.S05) with a pulsed ultraviolet He-Cd laser source at a $325 \mathrm{~nm}$ excitation wavelength.

The experimental procedures is follow scientific steps, there is:

1. Initialization of the plant extract: Matricaria flowers plant were collected, washed with water, dried and ground well.

2. Biosynthesis of silver nanoparticles: weighed about $200 \mathrm{gm}$ of the plant, then $100 \mathrm{ml}$ of ethanol was added to it, and left at room temperature for one day. After 24 hour the mixture was filtered by means of apiece of gauze and then $200 \mathrm{ml}$ of the filtrate was taken and $800 \mathrm{ml}$ of silver nitrate solution was added to it. It was placed in a closed bottle, and the color of the solution changed from light brown to dark brown, and this is evidence of the formation of silver nanoparticles. The change was evidence by the Fig. 1, $\boldsymbol{a}, \boldsymbol{b}$, because of excitation of surface plasmon vibrations.
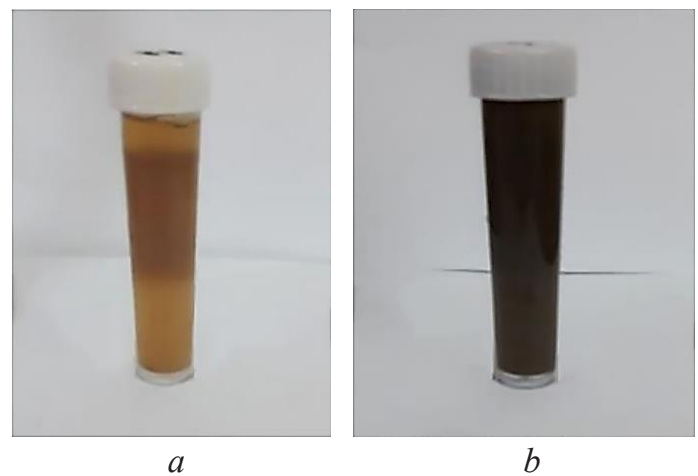

Fig. 1. Change the color of plant extract solution:

$a$-Matricaria flower extract solutions; $b$ - the formation of silver nanoparticles

It is possible to notice from Fig. 1 the change color of plant extract solution after adding silver nitrate to it

Where $a$ - represent the plant extract and $b$ - it represents the extract after adding silver nitrate to it and converting it to ananoscale Matricaria flower extract solutions after completion of the reaction in silver nanoparticles [7].

The inherent limitations in this study are include to use the type medium of plant to created silver nanoparticles where the ethanol medium is better than of water medium of plant.

3. Characterization: The structural analysis of the prepared silver nanoparticles has been carried out by X-ray diffractometer XRD (PANalytical unit using software X'Pert ${ }^{\circ}$ Pro3) equipped with $\mathrm{Cu}-\mathrm{K} \alpha$ radiation of wavelength $=1.5406 \mathrm{~A}^{\circ}$ at accelerating voltage $30 \mathrm{kV}$. The corresponding ligand coordination and functional groups of powders and prepared films were analyzed by FTIR Perkin Elmer Spectrum Version 10.4.00 FTIR Spectrophotometer in the region 500-4000 $\mathrm{cm}^{-1}$. The morphology and size distribution of AgNPs were investigated using S-3700N FESEM Field Emission Scaning Electron Microscopy (EESEM). The optical absorbance spectrum of colloidal solution was acquired using a Perkin-Elmer Lambda 11 UV/Vis Spectrophotometer. The Raman experiments were carried out using a Joibin-Ywon XplorE setup. The excitation laser beam $532 \mathrm{~nm}$ was focused onto the sample through the $100 \mathrm{X}$ lens of a confocal microscope.The laser power was $150 \mathrm{mw}$ and the Raman spectra were measured with an accumulation time of $15 \mathrm{~s}$. The photoluminescence measurements were carried out at room temperature using a PerkinlEmer spectrophotometer (L.S05) with a pulsed ultraviolet He-Cd laser source at a $325 \mathrm{~nm}$ excitation wavelength. 


\section{Results and discussion}

After the addition of $\mathrm{AgNO}_{3}$ to the extract plant solution of Matricaria flower, the color of the solution changes from highlight to brown indicating the formation of silver nanoparticles. The morphology and structural analysis of the prepared silver nanoparticles has been carried out by FESEM, XRD, FTIR. The optical absorbance spectra of silver colloidal solution was measured using. UV-visible spectrascopy. Raman scattering technique and photoluminescence experiments were carried out in order to study the optical emission of silver nanoparticles:

A. Field Emission Scanning Electron Microscopy (FESEM).

The FESEM measurement depicts the surfaces of prepared materials with high magnification and high resolution. It shows the particle size and shape of the sample used. The results of a field Emission Scanning electron microscopy revealed that the silver nanoparticles were oval and scattered on the surface of the licorice root extract, as well the emergence of large silver nanoparticles formed due to the gathering of small particles, as shown in the Fig. 2. It shows that the size of AgNPS (32.55-70.55) nm, with average size $45.98 \mathrm{~nm}$ in Matricaria flower plant.

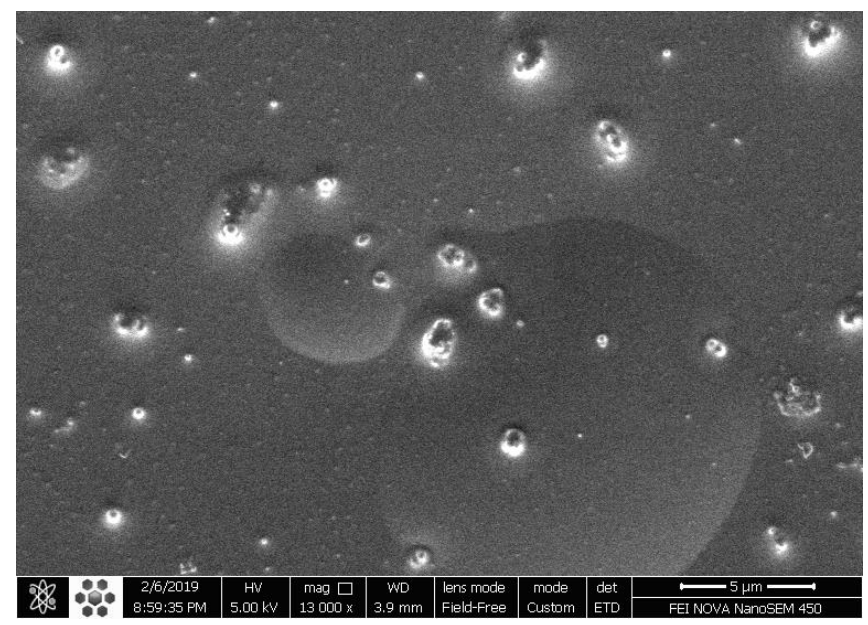

Fig. 2. FESEM image of silver nanoparticles

Uv-visible spectroscopy. Uv-visible spectroscopy of the plant extract was measured with a UV-1800 series spectrophotometer at are solution of $(0-1) \mathrm{nm}$. It is a widely used technique for characterization nanoparticles using matricaria flower extract.

The addition of Matricaria flowers extract to silver nitrate solution resulted in color change of the solution due to the production of silver nanoparticles the SPR of silver nanoparticles produced a peak centered near $350 \mathrm{~nm}$ for extract it was measured by UV-Vis spectrophotometer, the Fig. 3 shows that [11].

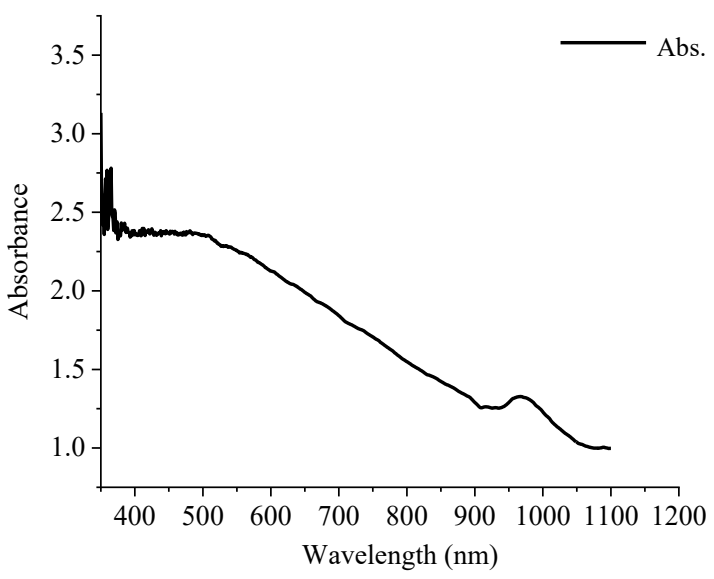

Fig. 3. Uv-visible spectrum of silver nanoparticles 
Fig. 3 shows Uv-visible spectra of silver nanoparticles which synthesized from the Matricaria Flowers extract.

FTIR analysis. FTIR spectra are used in the identification of biomolecules for capping and stabilizing the AgNPS. FTIR spectra of the silver nanoparticles given in the below Fig. 7 exhibited peaks at $3667.98 \mathrm{~cm}^{-1}, 3554.23 \mathrm{~cm}^{-1}, 3467.77 \mathrm{~cm}^{-1}, 1455.52 \mathrm{~cm}^{-1}$ and $1384.79 \mathrm{~cm}^{-1}$ result from stretching of the $\mathrm{O}-\mathrm{H}$ hydroxyl group, $\mathrm{C}=\mathrm{O}$ which reveals that the water soluble heterocyclic components, and $\mathrm{CH}_{3}$. Fig. 4 shows the FTIR analysis spectrum of Matricaria flowers extract showed in the Table 1.

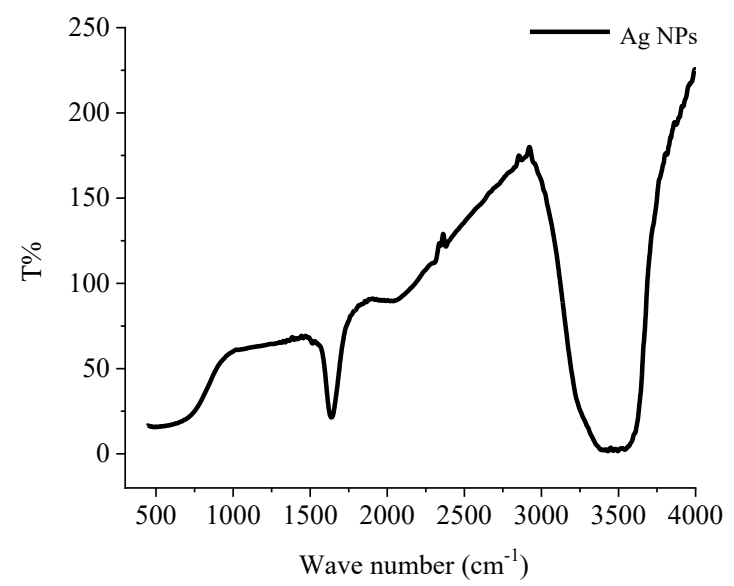

Fig. 4. FTIR spectrum of Matricaria Flowers extract

Table 1

The FTIR analysis information of Matricaria flowers extract

$\begin{array}{ccc}\text { Absorbance peak }\left(\mathbf{c m}^{-\mathbf{1}}\right) & \text { Functional groups } & \text { Plant name } \\ 3467.77,3554.23,3667.98 & \mathrm{O}-\mathrm{H} & \\ 1455.52 & \mathrm{C}=\mathrm{O} & \text { Matricaria flowers } \\ 1384.79 & \mathrm{CH} 3 & \end{array}$

Raman Scattering Technique. Raman microscopy system used in the current work is equipped with laser wavelengths at $532 \mathrm{~nm}$, it was used to higher quality spectra with a shorter time using $532 \mathrm{~nm}$ wavelength laser. Laser beam in the range of $0.1-1.5 \mathrm{~mW}$ was focused on a spot of diameter $3 \mu \mathrm{m}$. Raman spectrum excited with $532 \mathrm{~nm}$ laser line, Raman spectrum of silver nanoparticles was investigated at excitation wavelength the additional Raman peaks at about 1455, 3603, and $3557 \mathrm{~cm}^{-1}$ are found this is roughly identical to the FTIR spectra of the silver nanoparticles. The Raman spectra of silver nanoparticles were appear in Fig. $\mathbf{5}$ at room temperature.

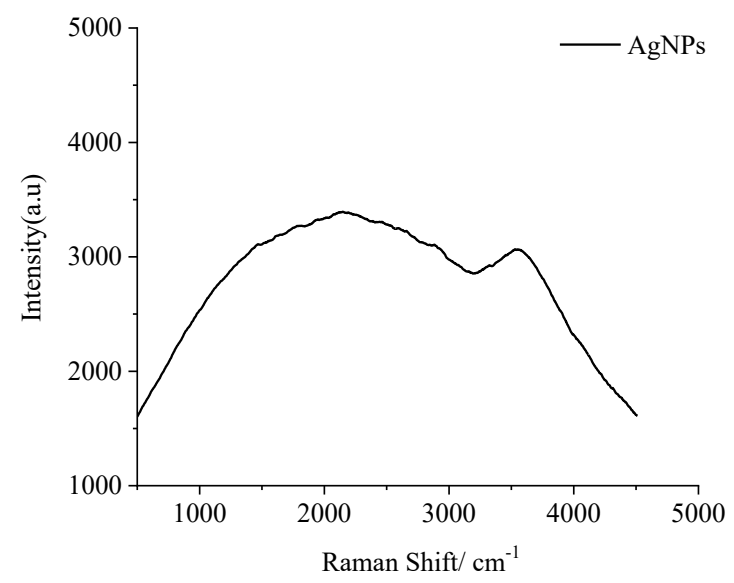

Fig. 5. Raman scattering spectra of silver nanoparticles at room temperature 
Study of Photoluminescence properties spectra. Photoluminescence is a prevalent spectroscopic technique to execute emission studies on metal particle systems. It shows emissions intensity about 200 to $900 \mathrm{~nm}$ as displayed in Fig. 6. Compound colloidal silver nanoparticles were found to be photoluminescence. Photoluminescence spectra obtained from the synthesized AgNPS were done at room temperature were shown in Fig. 6. It's clear from Fig. 6 the Photoluminescence emission peak and UV-visible peak appear at the same wave length.The visible luster of silver nanoparticles had been studied earlier and due to the excitation of the electron from occupied levels to states higher than the Fermi level [13]. The PL spectra of silver nanoparticles exhibit two emission bands: one is in the UV region $350 \mathrm{~nm}$ and the other is in the visible region $650 \mathrm{~nm}$.

Antibacterial activity of Matricaria Chamomilla flowers extract and silver nanoparticles.

The plants antibacterial activity and silver nanoparticles synthesized Matricaria flowers were completed by agar diffusion method. The inhibitory effect of AgNPS was studied using an alcoholic extract of Matricaria flowers against two types of bacteria (Escherichia Coli and Staphylococcus aurous). The maximum region of inhibition for extract against $E$. Coli is $11 \mathrm{~nm}$. And the maximum region of inhibition for silver nanoparticles is $12 \mathrm{~nm}$. The maximum region of inhibition for extract against Staphylococcus aurous is $9 \mathrm{~nm}$. And the maximum region of inhibition for silver nanoparticles is $11 \mathrm{~nm}$. Against Staphylococcus aurous en handing effectiveness of the Matricaria flowers due to the presence of a number of effective chemical compounds such as flavonoids, carboy drates, proteins, and others. The reason for the difference in the effect of bacterial with plant extracts is due to the difference in the chemical structural of the cell wall and the silver nanoparticles are also associated with the negative charges of the cell wall and its break down, as well as mutating proteins and then the death of the cell $[2,14]$.

Fig. 7 shows antibacterial activity of silver nanoparticles for both types of bacteria.

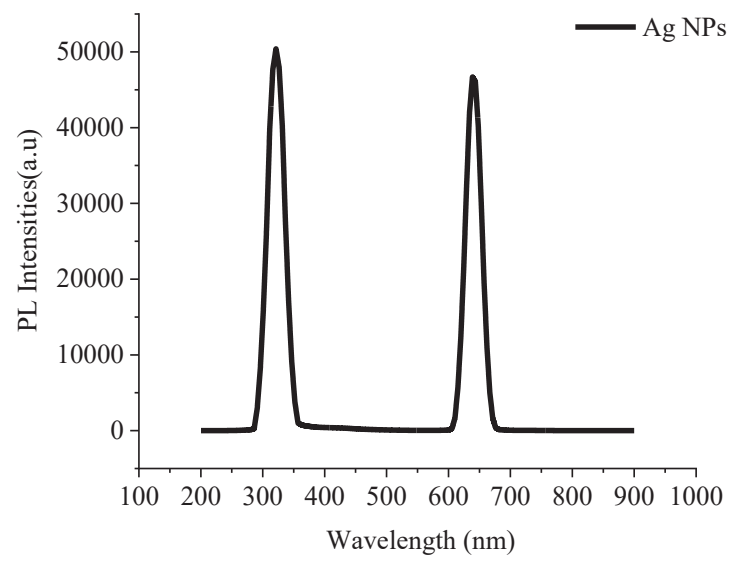

Fig. 6. Photoluminescence emission spectra of silver nanoparticles at room temperature
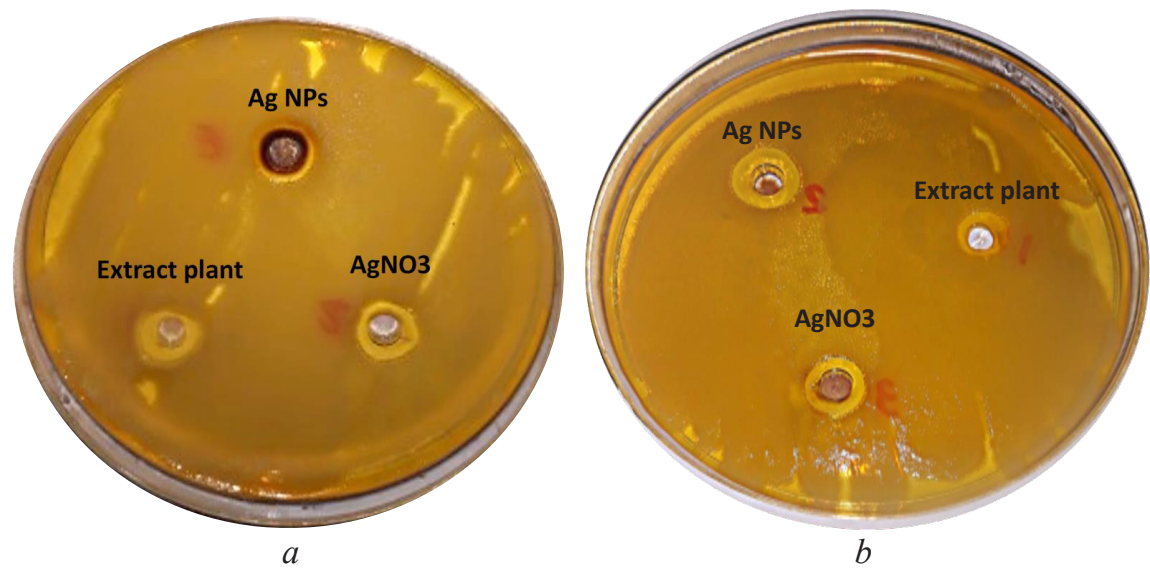

Fig. 7. Antibacterial activity of silver nanoparticles: $a$ - against of staphylococcus bacteria; $b$ - against of E.Coli bacteria 
Staphylococcus aurous bacteria appears more resistant for silver nano particles than Escherichia Coli bacteria due to the difference in the chemical structural of the cell wall.

\section{Conclusions}

Green synthesis technology of silver nanoparticles present an easy, efficient, clean, non-toxic and eco-friendly method. A different of plant extracts can be used for the biosynthesis of silver nanoparticles and other metals nanoparticles. An easy green synthesis of silver nanoparticles using Matricaria flowers extract at room temperature was successfully synthesized. Easy green approach for the synthesis supplying a cost efficient and an effective way for the synthesis of silver nanoparticles.

The optical properties were studied in the wavelength range of (200-900) nm. These results suitable to be use the silver nanoparticles in sensors and many electronic, electrical and medical applications. In this work, it is noticed that the PL spectra of silver nanoparticles exhibit two emission bands: one is in the UV region $350 \mathrm{~nm}$ and the other is in the visible region $650 \mathrm{~nm}$ which are related to the surface plasmon resonance properties. This is roughly identical to the absorption spectrum results. Raman spectroscopy measurements of Ag showed a strong band located at 1455,3603 , and $3557 \mathrm{~cm}^{-1}$ this is roughly identical to the FTIR spectra of the silver nanoparticles. Let's expect these results will useful trial to use metal-enhanced Photoluminescence or Raman scattering spectra in different application such as bio sensing and opto electronics. The synthesized silver nanoparticles present antibacterial activity antimicrobial activities against some kinds of bacteria such as Escherichia coli and Staphylococcus aurous. Antibacterial activity of silver nanoparticles shows the resistant of Staphylococcus aurous bacteria for inhibition than the Escherichia Coli bacteria due to the difference in the chemical structural of the cell wall. Advantages of utilizing green synthesis is that it is energy effective, cost efficient, saving human health and surroundings. This green synthesis method could be considered instead of classical chemical and physical methods and therefore has a potential applications in many field such as biomedical and medical applications.

\section{Acknowledgments}

The authors have no funding from any professional official institution. The research was funded at the self-expense of the authors.

\section{References}

[1] Jassim, N. M., Kareem, N. A., Ibrahim, N. I. (2021). Investigation on synthesis, structural and nonlinear optical responses of cadmium selenide coated with gold nanoparticles induced by femtosecond laser excitation. Eastern-European Journal of Enterprise Technologies, 3 (5 (111)), 13-18. doi: https://doi.org/10.15587/1729-4061.2021.231422

[2] Ali, A. H., Mubarak, T. H., Jassim, N. M., Jasim, I. M. (2019). Biosynthesis and study of some optical properties of silver nanoparticles in corporate plasmon resonance for antibacterial activities. Plant Archives, 19, 1063-1066. Available at: http:// www.plantarchives.org/SPL\%20ISSUE\%20SUPP\%202,2019/188\%20(1063-1066).pdf

[3] Farhan, A. M., Jassim, R. A., Kadhim, N. J., Mehdi, W. A., Mehde, A. A. (2017). Baghdad Science Journal Synthesis of Silver Nanoparticles from Malva parviflora Extract and Effect on Ecto-5'-Nucleotidase(5'-NT), ADA and AMPDA Enzymes in Sera of Patients with Arthrosclerosis. Baghdad Science Journal, 14 (4), 742-750. doi: https://oi.org/10.21123/ bsj.14.4.742-750

[4] Fathima, R., Mujeeb, A. (2021). Plasmon enhanced linear and nonlinear optical properties of natural curcumin dye with silver nanoparticles. Dyes and Pigments, 189, 109256. doi: https://doi.org/10.1016/j.dyepig.2021.109256

[5] Bahrulolum, H., Nooraei, S., Javanshir, N., Tarrahimofrad, H., Mirbagheri, V. S., Easton, A. J., Ahmadian, G. (2021). Green synthesis of metal nanoparticles using microorganisms and their application in the agrifood sector. Journal of Nanobiotechnology, 19 (1). doi: https://doi.org/10.1186/s12951-021-00834-3

[6] Sabir, S., Arshad, M., Chaudhari, S. K. (2014). Zinc Oxide Nanoparticles for Revolutionizing Agriculture: Synthesis and Applications. The Scientific World Journal, 2014, 1-8. doi: https://doi.org/10.1155/2014/925494

[7] Alshehri, A. A., Malik, M. A. (2020). Phytomediated Photo-Induced Green Synthesis of Silver Nanoparticles Using Matricaria chamomilla L. and Its Catalytic Activity against Rhodamine B. Biomolecules, 10 (12), 1604. doi: https://doi.org/ 10.3390/biom10121604 
[8] Uddin, I., Ahmad, K., Khan, A. A., Kazmi, M. A. (2017). Synthesis of silver nanoparticles using Matricaria recutita (Babunah) plant extract and its study as mercury ions sensor. Sensing and Bio-Sensing Research, 16, 62-67. doi: https://doi.org/ 10.1016/j.sbsr.2017.11.005

[9] Mladenova, B., Diankov, S., Karsheva, M., Stankov, S., Hinkov, I. (2018). Plant mediated synthesis of silver nanoparticles using extracts from Tilia cordata, Matricaria chamomilla, Calendula officinalis and Lavandula angustifolia flowers. Journal of Chemical Technology and Metallurgy, 53 (4), 623-630. Available at: https://dl.uctm.edu/journal/node/j2018-4/ 1_18-37_p_623-630.pdf

[10] Dhafer, C. E. B., Mezni, A., Smiri, L. S. (2017). Surface-enhanced Raman scattering study of Ag-PVP interactions in the biocompatible Ag@PVP nanoparticles. Journal of the Tunisian Chemical Society, 19, 152-157. Available at: https://www. researchgate.net/profile/Amine-Mezni/publication/324792913_Surface-enhanced_Raman_scattering_study_of_Ag-PVP_ interactions_in_the_biocompatible_AgPVP_nanoparticles/links/5ae291deaca272fdaf $8 \mathrm{fb} 8 \mathrm{ec} /$ Surface-enhanced-Ramanscattering-study-of-Ag-PVP-interactions-in-the-biocompatible-AgPVP-nanoparticles.pdf

[11] Naikoo, G. A., Mustaqeem, M., Hassan, I. U., Awan, T., Arshad, F., Salim, H., Qurashi, A. (2021). Bioinspired and green synthesis of nanoparticles from plant extracts with antiviral and antimicrobial properties: A critical review. Journal of Saudi Chemical Society, 25 (9), 101304. doi: https://doi.org/10.1016/j.jscs.2021.101304

[12] Kim, D., Jang, D. (2007). Synthesis of nanoparticles and suspensions by pulsed laser ablation of microparticles in liquid. Applied Surface Science, 253 (19), 8045-8049. doi: https://doi.org/10.1016/j.apsusc.2007.02.153

[13] Singh, A., Gautam, P. K., Verma, A., Singh, V., Shivapriya, P. M., Shivalkar, S. et. al. (2020). Green synthesis of metallic nanoparticles as effective alternatives to treat antibiotics resistant bacterial infections: A review. Biotechnology Reports, 25, e00427. doi: https://doi.org/10.1016/j.btre.2020.e00427

[14] Ali, M. A., Ahmed, T., Wu, W., Hossain, A., Hafeez, R., Islam Masum, M. M. et. al. (2020). Advancements in Plant and Microbe-Based Synthesis of Metallic Nanoparticles and Their Antimicrobial Activity against Plant Pathogens. Nanomaterials, 10 (6), 1146. doi: https://doi.org/10.3390/nano10061146

Received date 07.07.2021

Accepted date 30.10.2021

Published date 18.11.2021
(C) The Author(s) 2021

This is an open access article under the Creative Commons CC BY license

How to cite: Jassim, N. M., Ali, A. H., Mohammed, A. J. (2021). Experimental implementation of Raman scattering spectroscopy, photoluminescence and some optical properties of silver nanoparticles created by eco-friendly technique. EUREKA: Physics and Engineering, 6, 3-10. doi: https://doi.org/10.21303/2461-4262.2021.002147 